\title{
Pharmacological interventions for pain for life-limiting conditions in children and adolescents (Protocol)
}

Beecham E, Howard R, McCulloch R, Candy B, Laddie J, Rees H, Vickerstaff V, BluebondLangner M, Jones $L$
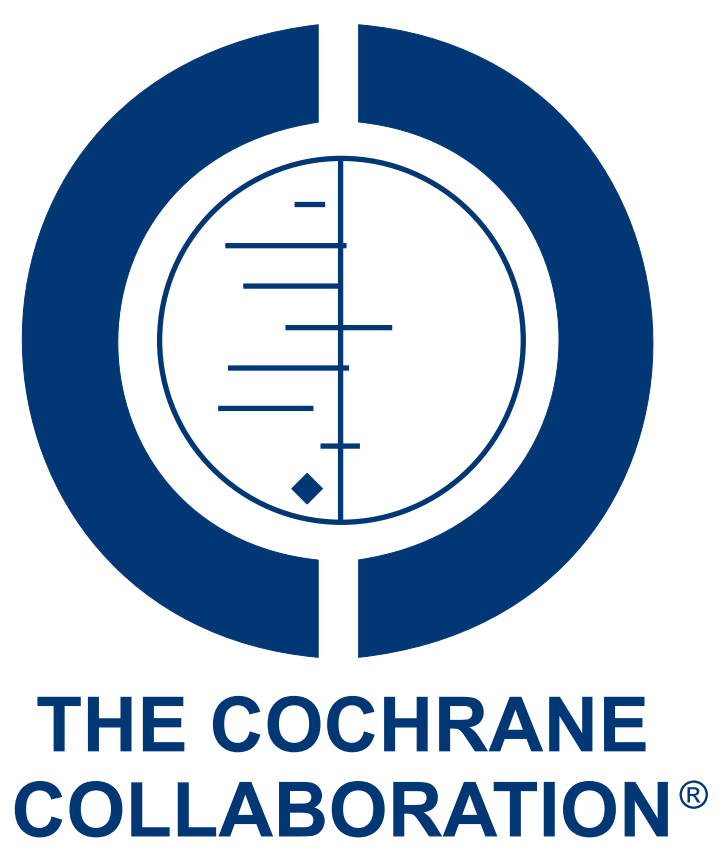

This is a reprint of a Cochrane protocol, prepared and maintained by The Cochrane Collaboration and published in The Cochrane Library 2013, Issue 9

http://www.thecochranelibrary.com

\section{WILEY}

Pharmacological interventions for pain for life-limiting conditions in children and adolescents (Protocol)

Copyright $\odot 2013$ The Cochrane Collaboration. Published by John Wiley \& Sons, Ltd. 
TABLE OF CONTENTS

HEADER . . . . . . . . . . . . . . . . . . . . . . . . . . . . . . . . . . . . 1

ABSTRACT . . . . . . . . . . . . . . . . . . . . . . . . . . . . . . . . . . . . . . . 1

BACKGROUND . . . . . . . . . . . . . . . . . . . . . . . . . . . . . . . . . . . .

OBJECTIVES . . . . . . . . . . . . . . . . . . . . . . . . . . . . . . . . . . . . . . .

METHODS . . . . . . . . . . . . . . . . . . . . . . . . . . . . . . . . . . . . . . . .

ACKNOWLEDGEMENTS . . . . . . . . . . . . . . . . . . . . . . . . . . . . . . . . . .

REFERENCES . . . . . . . . . . . . . . . . . . . . . . . . . . . . . . . . . . . . . . 6

APPENDICES . . . . . . . . . . . . . . . . . . . . . . . . . . . . . . . . . . . . . 8

CONTRIBUTIONS OF AUTHORS . . . . . . . . . . . . . . . . . . . . . . . . . . . . . . . . . . . . . . . 9.9

DECLARATIONS OF INTEREST . . . . . . . . . . . . . . . . . . . . . . . . . . . . . . . . . 10

SOURCES OF SUPPORT . . . . . . . . . . . . . . . . . . . . . . . . . . . . . . . . . . . . . . . . .

Pharmacological interventions for pain for life-limiting conditions in children and adolescents (Protocol)

Copyright $\odot 2013$ The Cochrane Collaboration. Published by John Wiley \& Sons, Ltd. 


\title{
[Intervention Protocol]
}

\section{Pharmacological interventions for pain for life-limiting conditions in children and adolescents}

\author{
Emma Beecham ${ }^{1,2}$, Richard Howard ${ }^{3}$, Renée McCulloch ${ }^{4}$, Bridget Candy ${ }^{1}$, Jo Laddie ${ }^{5}$, Henrietta Rees ${ }^{2}$, Victoria Vickerstaff ${ }^{1}$, Myra $^{2}$ \\ Bluebond-Langner ${ }^{2,6}$, Louise Jones ${ }^{1}$ \\ ${ }^{1}$ Marie Curie Palliative Care Research Unit, UCL Mental Health Sciences Unit, London, UK. ${ }^{2}$ Louis Dundas Centre for Children's \\ Palliative Care, UCL Institute of Child Health, London, UK. ${ }^{3}$ Department of Anaesthesia, Great Ormond Street Hospital, London, UK. \\ ${ }^{4}$ Louis Dundas Centre for Children's Palliative Care, Palliative Care Team, Great Ormond Street Hospital, London, UK. ${ }^{5}$ Department \\ of Paediatric Palliative Medicine, Evelina London Children's Hospital, London, UK. ${ }^{6}$ Department of Sociology, Anthropology and \\ Criminal Justice, Rutgers University, Camden, New Jersey, USA \\ Contact address: Emma Beecham, e.beecham@ucl.ac.uk.
}

Editorial group: Cochrane Pain, Palliative and Supportive Care Group.

Publication status and date: New, published in Issue 9, 2013.

Citation: Beecham E, Howard R, McCulloch R, Candy B, Laddie J, Rees H, Vickerstaff V, Bluebond-Langner M, Jones L. Pharmacological interventions for pain for life-limiting conditions in children and adolescents. Cochrane Database of Systematic Reviews 2013, Issue 9. Art. No.: CD010750. DOI: 10.1002/14651858.CD010750.

Copyright (C) 2013 The Cochrane Collaboration. Published by John Wiley \& Sons, Ltd.

\begin{abstract}
A B S T R A C T
This is the protocol for a review and there is no abstract. The objectives are as follows:

To evaluate the evidence on the effectiveness of different pharmacological interventions used for pain in CYP with LLC.
\end{abstract}

\section{B A C K G R O U N D}

\section{Description of the condition}

Pain is one of the most common symptoms in children and young people (CYP) with life-limiting conditions (LLCs) (Beretta 2010; Bradshaw 2005; Feudtner 2011; Goldman 2006; Hechler 2008; Jalmsell 2006; Wolfe 2000). In this review, LLC refers to 'any condition from which there is no reasonable hope of cure and from which the child or young adult will die prematurely' and life-threatening conditions are defined as 'those for which curative treatment may be feasible but can fail' (ACT 2009). LLCs are seen to be rising in the UK (Fraser 2012), with 32 per 10,000 children having an LLC. Sources of pain in this population include on-going tissue damage due to pathological processes, recurrent injury, therapy and invasive diagnostic or therapeutic procedures. Increasing evidence suggests that pain is not well managed in such children, especially towards the end of life. In a large cross-sectional study of children with cancer deemed 'palliative', Goldman 2006 found that $91.5 \%$ of the 164 children in the study experienced pain in the month before death. Beretta 2010 found pain to be the most frequent symptom, with $87 \%$ of their sample of 47 children with cancer experiencing pain during the 'end stage'. Drake 2003 found that in a sample of $30,53 \%$ of 'dying' children experienced pain in the last week of their lives.

\section{Types of pain}


Pain can be characterised in several ways, individually or in combination: by mechanism or pathophysiology, by intensity, by temporality, or by location.

1. Pain mechanisms

Two basic pain mechanisms are known: nociceptive and neuropathic. Nociceptive pain occurs as the result of tissue damage and/ or inflammation due to physical, chemical or thermal injury (e.g. traumatic or ischaemic pain, arthritis, muscle spasm, mucositis, gastritis, or other visceral inflammatory processes). Neuropathic pain occurs when a lesion of the central or peripheral nervous system causes nociceptive dysfunction (IASP 2012) (e.g. from direct tumour invasion or neural toxicity from chemotherapy or infection). Nociceptive and neuropathic pain can occur separately or together in the same individual. The importance of distinguishing between these two mechanisms is that analgesics are developed for action on specific mechanisms, and so outcome can vary depending on the type of pain.

2. Intensity

Pain intensity is usually measured on a scale of 0 to 10 , or 0 to 100 , using a linear visual analogue scale (VAS) or other pain intensity 'measurement tool' such as the Wong-Baker Faces Scale (Wong 1988). Intensity can also be described using the four-point categorical pain intensity scale with corresponding wording, 'none, mild, moderate, severe', or as characterised in the World Health Organisation (WHO) two-step pain management algorithm: mild VAS (4 to 6), moderate (VAS 7 to 8 ) or severe (VAS 9 to 10), which recommends pharmacological interventions of increasing potency to be used for mild and moderate to severe pain (WHO 2012). The current version of the WHO document differs from the original three-step ladder, which previously included a middle step of using a 'weaker' opioid for moderate pain before a 'stronger' opioid in the third step (WHO 1996). Pain intensity measurement is potentially complex in children who are too young/preverbal or non-verbal and unable to describe or quantify their pain. The literature on this subject is enormous, and a large number of pain measurement tools suitable for children of different ages in a variety of settings have been devised (Stinson 2006; Von Baeyer 2009). However, no definitive tools adequately measure persistent pain in children with palliative care needs. It is important to recognise that in clinical studies, the accuracy and reliability of such tools depend on their validity for use in the situation described (see also Secondary outcomes).

3. Temporality

Pain can be described by its temporality, although as is emphasised in the WHO guidelines, temporality does not define treatment strategies. Pain may be experienced and described as acute pain (< 30 days), chronic pain ( $>3$ months, with behaviours in response to pain that do not remit; Hain 2011), persistent pain (covers 'long-term pain related to medical illness'), episodic or recurrent pain ('occurs intermittently over a long period of time and the child can be pain free in between each painful episode'), breakthrough pain ('temporary increase in the severity of pain over and above the pre-existing baseline pain level' and can be predictable or unpredictable with/without an identifiable cause), incident pain (from an identifiable cause) and 'end of dose' pain (occurs before a scheduled dose of an around-the-clock analgesic) (WHO 2012). 4. Location

Pain is sometimes characterised by location in the body (e.g. bone pain, headache, abdominal pain or musculoskeletal pain).

\section{Description of the intervention}

For the purpose of this review, we will focus on pharmacological interventions for the relief of pain causally related to the LLC (disease-related pain). Pain due to diagnostic and therapeutic procedures and postoperative pain will be excluded.

We shall assess evidence on the effectiveness of pharmacological interventions, using a framework adapted from WHO guidelines for pain management in children with medical conditions (WHO 2012). These include the following:

1. Non-opioid analgesics such as paracetamol and non-steroidal anti-inflammatory drugs (NSAIDs).

2. Opioids such as morphine, methadone, hydromorphone, buprenorphine, codeine, fentanyl and oxycodone.

3. Local anaesthetics such as lidocaine, bupivacaine and levo bupivacaine.

4. 'Adjuvant analgesics'. This group includes all drugs given for pain whose primary indication is not analgesia (e.g. most drugs commonly used for neuropathic pain, such as tricyclic antidepressants (TCAs), selective serotonin reuptake inhibitors (SSRIs), serotonin-norepinephrine reuptake inhibitors (SNRIs), anticonvulsants such as gabapentin and carbamazepine). Muscle relaxants and antispasmodics such as baclofen and hyoscine, steroids, the adrenergic analgesic clonidine and the $\mathrm{N}$-methyl-D-aspartate (NMDA) antagonists ketamine and dexmedetomidine are also included in this category.

\section{How the intervention might work}

Pharmacological interventions used to treat pain in CYP with LLC are numerous and varied; they work in different and complex ways, with some mechanisms of action still poorly understood. We will briefly consider the mechanisms of action according to the above groupings, giving examples from each group, recognising that these interventions may be used for pain from a variety of causes occurring in a variety of temporalities and in a range of clinical conditions as defined by the International Classification of Diseases and Related Health Problems, Tenth Revision (ICD10), codes.

1. Non-opioids

Non-opioid analgesics traditionally include paracetamol and the NSAIDs (e.g. ibuprofen, diclofenac, ketorolac). Some of these analgesics, such as ketorolac and diclofenac, are still of uncertain 
potency. Paracetamol is an analgesic and antipyretic and is probably the most popular simple analgesic used in children for pain of mild to moderate intensity (Anderson 2008). Paracetamol has numerous putative mechanisms of analgesia, such as inhibiting prostaglandin synthesis within the CNS (cyclo-oxygenase (COX)3 , COX-2b), blocking impulse generation within the bradykininsensitive chemoreceptors responsible for the generation of nociceptive impulses and antagonising NMDA (Jacqz-Aigrain 2006). The recommended oral dosage starts at $20 \mathrm{mg} / \mathrm{kg}$ as a single dose, then 10 to $15 \mathrm{mg} / \mathrm{kg}$ every 8 to 12 hours for neonates up to 500 mg; $1 \mathrm{~g}$ every 4 to 6 hours for 16- to 18-year-olds (BNF 2012). NSAIDs are a diverse group of drugs that share similar antipyretic, analgesic and anti-inflammatory effects but may show different response characteristics (Jacqz-Aigrain 2006). Ibuprofen, for example, is a propionic acid derivative and a non-selective cyclooxygenase inhibitor, and its recommended dosage is from $5 \mathrm{mg}$ per kilogram for infants aged 1 to 3 months up to 300 to $400 \mathrm{mg}$ for children 12 to 18 years old, 3 to 4 times daily by mouth (BNF 2012).

2. Opioids

Numerous opioids are used to relieve pain in CYP with LLC, including (but not limited to) morphine, codeine, buprenorphine, fentanyl, methadone and oxycodone. Opioids bind to specific receptors found principally in the central nervous system and the gastrointestinal tract. Morphine is widely regarded as the first-line major opioid in CYP with LLC experiencing severe pain. Morphine acts directly on opioid receptors, and a principal metabolite morphine-6-glucuronide (M6G) also has analgesic activity. Opioids can cause constipation and itch, as well as serious side effects such as extreme somnolence and depression of respiration, particularly when used in excess in opioid-naive individuals and young infants. Although age-related changes in the pharmacokinetics of opioids are still not well understood, it is known that most agerelated changes are more apparent in the first year of life (as the result of pharmacokinetic differences, particularly reduced renal clearance in the first few months of life), and from then on, the ability of children to metabolise opioids seems similar to that of adults (Ballentine 2012). Total body morphine clearance is $80 \%$ of adult values by 6 months of age (Bouwmeester 2004). However, it has been shown that M6G may have faster renal clearance in children, and therefore children may actually need higher doses given at smaller intervals than in adults (Mashayekhi 2009). Data on the use of opioids in children with LLC are still lacking (Zernikow 2009), and on-going debate requires further study to provide conclusive evidence.

3. Local anaesthetics

Local anaesthetics are ion channel $(\mathrm{Na}+)$ blocking drugs that can treat and prevent all types of pain by blocking nociceptive pathways and suppressing nociceptor excitability. They are normally given by injection close to nerves peripherally or centrally (intrathecal or epidural), but topical preparations, including a lowdose transdermal patch formulation that is effective for some types of neuropathic pain, are also available. Local anaesthetics in clinical use include the amides lidocaine, bupivacaine and levo bupivacaine, and the esters benzocaine, tetracaine and chloroprocaine. 4. Adjuvants

Adjuvants of interest in this review are drugs whose primary indication is not for pain but that nevertheless have analgesic properties. Examples of adjuvants for neuropathic pain include some anticonvulsants, antidepressants, steroids and the NMDA antagonist ketamine. Skeletal muscle relaxants, such as baclofen, and antispasmodics, such as hyoscine, are sometimes given for pain. Adjuvants make up a varied group and work in many different ways. In this review, we will consider only adjuvants that are explicitly administered for pain relief.

\section{Routes of administration}

In CYP, the preferred route, where possible, is oral because it is the simplest, most effective and least painful (WHO 2012). However, other routes are frequently necessary because of varying clinical needs. Examples include buccal, rectal, transdermal, intramuscular, subcutaneous, intravenous, epidural and intrathecal routes.

\section{Why it is important to do this review}

The evidence base that is currently available to guide clinical practice in this area of pain management in CYP with LLC is limited, and whilst some clinical reviews have been published, no systematic review of the international literature has been performed to date. A recent survey conducted by the Association for Paediatric Palliative Medicine (APPM) found that clinicians have an urgent need for systematic review evidence to support their prescribing (Brook 2012).

\section{O B J E C T I VES}

To evaluate the evidence on the effectiveness of different pharmacological interventions used for pain in CYP with LLC.

\section{METHO D S}

\section{Criteria for considering studies for this review}

\section{Types of studies}

We will include randomised controlled trials (RCTs) (including cluster RCTs and cross-over trials), quasi-randomised studies, $\mathrm{n}$ of 1 studies, studies that are not randomised but include a clearly 
defined comparator group and time series analyses that have investigated pharmacological treatments for pain associated with LLC in CYP.

\section{Types of participants}

CYP aged 0 to 18 years (under the age of 19), of either sex, with an LLC. When screening the papers, the review authors (EB, JL, HR) will determine whether a condition is life-limiting by using the Richard Hain Dictionary (Hain 2010) of ICD-10 diagnoses that have been judged by professionals working in paediatric palliative care to be life-limiting, and that were recently used in a paper plotting the national prevalence of LLC in this population (Fraser 2012). They can be broken down into the following groups: infections; leukaemia; other malignant neoplasms; other neoplasms; sickle cell disorders; thalassaemia and other anaemias; other diseases of blood and blood-forming organs; cystic fibrosis; other endocrine, nutritional and metabolic disorders; epilepsy; cerebral palsy and other paralytic syndromes; other disorders of the nervous system; diseases of the circulatory system; diseases of the respiratory system; diseases of the musculoskeletal system and connective tissue; diseases of the genitourinary system; conditions originating in the perinatal period; congenital anomalies and other causes; and non-malignant haematological disorders (Cochrane 2007). CYP who have pain related to their LLC will be included. Setting: all settings, including home, hospital, hospice and residential school.

\section{Types of interventions}

Interventions will include any pharmacological intervention given at any dose for any time period on its own or in combination, or with a control or comparator group (see below). We will exclude studies on non-pharmacological interventions.

Control or comparator groups will include any other pharmacological interventions; psychological interventions such as relaxation, hypnosis and cognitive behavioural therapy; placebo; and alternative dosing regimens or routes of administration.

\section{Types of outcome measures}

For all outcome measures, we will report on the mechanisms of reporting pain in this population, which commonly features preverbal and non-verbal children, and will take into consideration in our own results the types of outcome measures used (e.g. observational/proxy/self-report).

\section{Primary outcomes}

Primary outcomes will be pain control and adverse events. Pain control will be measured by changes in pain intensity scales; other indicators such as changes in physiological parameters may also be used (baseline or final value scores at end of follow-up) and will include both continuous and dichotomous pain outcomes. We will report what each paper suggests as an adequate reduction of pain/period of maintenance of pain reduction and will synthesise findings accordingly; however, as advised in the 'Authoring or Assessing a Cochrane Protocol, Review, or Review Update for the PaPaS Review Group' guidance, we will include in our metaanalysis only studies that use moderate or greater pain as baseline (Cochrane 2011). To facilitate the review process, all forms of pain measurement in children, both validated and non-validated, will be considered during the review process. We shall report data on all adverse events identified.

\section{Secondary outcomes}

As the effectiveness of analgesia is also measured in terms of changes in physical and psychological functioning and well-being (McGrath 2008), we will include assessments using validated instruments, psychological or social measures such as mental health status and functioning scales, quality of life, well-being and quality of care scales for children, such as the Pediatric Quality of Life Inventory $^{T M}$ (PedsQL) (Varni 1999) and European Quality of Life 5-Dimensions (EQ-5D) (Ravens-Sieberer 2010) for their family. Health service use, including length of stay and number of hospital admissions, will be reviewed.

\section{Search methods for identification of studies}

We will use a combination of indexed and free-text terms to reflect the concepts of 'pharmacological intervention', 'CYP' and 'pain'. The LLC element will be identified during screening of papers. We will modify the search terms according to the constraints of each database. Please see Appendix 1 for the MEDLINE search strategy used.

\section{Electronic searches}

The following electronic databases will be searched: CENTRAL (on The Cochrane Library), MEDLINE, EMBASE, PsycINFO (all via Ovid SP) and CINAHL (via EBSCO host). No language restrictions will be applied.

\section{Searching other resources}

We will undertake the following additional search strategies:

1. Conversations with colleagues or key authors, or review of papers that they recommend

2. Contact with key authors who have published in this field

3. Conference proceedings where available, such as the International Symposium on Paediatric Pain

4. Internet searches

5. Forward and backward citation searches of included studies 
6. Handsearching of key journals (including Journal of Pain and Symptom Management)

\section{Data collection and analysis}

\section{Selection of studies}

Two review authors (EB, JL) will screen abstracts of all identified studies against the inclusion criteria. A third member of the review team (HR) will screen a sample of the abstracts to further validate the process. We will retrieve all possibly relevant articles in full text for assessment against the inclusion criteria. We have links to researchers with many different languages within University College London (UCL) and so will be able to translate many non-English studies; for those studies for which we do not have an in-house translator available, we will find an external translator. Differences in study selection between review authors will be resolved by discussion until consensus can be reached, or by consultation with a third party (HR). We plan to include a PRISMA study flow diagram in the full review (Liberati 2009) to document the screening process, as recommended in Part 2, Section 11.2.1, of the Cochrane Handbook on Systematic Reviews of Interventions (Higgins 2011a).

\section{Data extraction and management}

Two review authors (EB, JL) will start to independently extract the data using standardised data extraction forms developed by the review authors. If necessary, in cases of disagreement or discrepancy, data will be reviewed by a third review author (HR). When review authors have reached agreement on the types of information to be extracted after they have reviewed a significant proportion of the papers, one review author (EB) will continue to extract the data from the remaining papers. Where possible, the following information will be obtained for each study.

1. The number of patients eligible, the number of participants randomly assigned, and reasons why patients were not included in the trial.

2. The number of participants evaluated at follow-up $(s)$ and what the follow-up time points were.

3. Participant demographics, including age, sex, diagnosis, ICD-10 code and type of healthcare setting (hospital/hospice/ home/residential school).

4. Trial design features on masking, whether parallel group or cross-over, features of randomisation, and sample size calculation.

5. Any necessary additional data on trial design and outcomes to allow completion of The Cochrane Collaboration's tool for assessing risk of bias.

6. Comparison interventions, including duration and mode.
7. Outcome data on pain reduction at all time points, including how outcome was measured, and mean or categorical scores of the main outcome and other outcomes.

8. Adverse effects.

9. Comment on the success of blinding (of researchers and participants) given the possibility of side effects unblinding participants.

10. Drop-out rates and reasons why.

11. Concurrent use of other drugs, including analgesics, and exclusions.

12. Quality of life of CYP and family and how this was measured.

13. Other behavioural and psycho/social measures and the scales used to measure them.

In cases where information is lacking, we will attempt to make contact with trial authors or trial sponsors.

\section{Assessment of risk of bias in included studies}

We will assess and report on the risk of bias of included RCTs using The Cochrane Collaboration's tool for assessing risk of bias (Higgins 2011a). This recommends explicit reporting of the following quality elements for RCTs: sequence generation; allocation concealment; blinding; completeness of outcome data; and selective outcome reporting. For each quality domain, we will assess whether the risk of bias was low (if the study matched the criteria), high (if the study did not match the criteria) or unclear (if underreporting was noted). We will define trials as having an overall low risk of bias if they score a low risk of bias on four of the five domains in the risk of bias table. We will label a trial as having an unclear risk of bias if the trial provided too few details to allow a judgement of 'high' or 'low' risk of bias. One review author (EB) will assess the risk of bias of included studies; one review author (JL or HR) will check a significant proportion of papers, and disagreements will be resolved by discussion. Where needed, we will contact study authors to ask for additional information. We will incorporate the results of the risk of bias assessment into the review through systematic narrative description and commentary about each item, leading to an overall assessment of the risk of bias of included studies and a judgement about the internal validity of the results of the review.

\section{Measures of treatment effect}

The null hypothesis to be tested is that, for the primary outcomes examined, the pharmacological interventions have no effect compared with placebo/other interventions. For dichotomous outcomes, we will calculate the risk ratio with $95 \%$ confidence interval (CI), and for continuous data, we will estimate the mean difference with $95 \%$ CI. Our reporting of these data is reliant on presentation by study authors of relevant data to allow us to calculate these statistics. To estimate the statistical significance of the results, we will calculate the $95 \%$ CI for each item. 


\section{Unit of analysis issues}

In the event that we identify a trial using a cluster design (in which participants were randomly assigned at group level), we will use the intracluster correlation coefficient (ICC) to estimate the effective sample size. If cross-over trials are to be included in a meta-analysis, statistical advice will be sought.

\section{Dealing with missing data}

If doubts arise about missing data (participant drop-outs, etc.), we will contact the study authors to obtain further information. When we are unable to obtain data, we will state it. When dichotomous data are missing and it has been possible to do an analysis, we will undertake a 'Sensitivity to missing data' analysis. We will address the potential impact of missing data on our findings in the 'Discussion' section of the review.

\section{Assessment of heterogeneity}

If a meta-analysis is conducted, we will use the $\mathrm{Chi}^{2}$ test and the $\mathrm{I}^{2}$ statistic to evaluate heterogeneity between trials (Higgins 2011b). A Chi ${ }^{2}$ test $P$ value of less than 0.10 or an $I^{2}$ value equal to or greater than $50 \%$ will be considered indicative of substantial heterogeneity.

\section{Assessment of reporting biases}

If a sufficient number of studies are identified and a meta-analysis is possible, we plan to assess publication bias by using funnel plots. However, funnel plots can be used only when a sufficient number of studies are included in the review, as use of funnel plots with insufficient numbers may lead the reader to the wrong conclusion that an asymmetrical funnel plot is caused by publication bias, or vice versa.

\section{Data synthesis}

For this review, we will first categorise the studies according to whether they consider nociceptive pain, neuropathic pain or both.
We will then group identified evidence by the different pharmacological interventions used (i.e. non-opioids, opioids, local anaesthetics and adjuvants). We will consider how they are used to treat the different types of pain, as well as the types of pain experienced by which condition, as categorised by the ICD-10 classification. If necessary, we will group and analyse results according to diagnosis and extent of pain, if this proves to be a more meaningful strategy. If there are sufficient trials by class of treatment we will combine statistically the data across trials. This will only be undertaken if the trials are sufficiently similar in measurement and population and are of sufficient quality. A fixed-effect (FE) model will be used in the first instance. If no substantial heterogeneity was noted, a random-effects (RE) model will be used to check the robustness of the FE model. If substantial statistical heterogeneity was observed, the RE model will be used a priori.

\section{Subgroup analysis and investigation of heterogeneity}

If a sufficient number of trials are combined in a meta-analysis and heterogeneity is identified between trials, subgroup analysis of the different diagnoses (according to ICD-10 code classification) will be undertaken.

\section{Sensitivity analysis}

We will use the GRADE system (Schunemann 2008) to assess the quality of the evidence associated with specific outcomes (e.g. pain reduction, quality of life improvement, adverse effects) and will construct a 'Summary of findings' table using the GRADE software. Through this approach, the body of evidence will be assessed as 'high', 'moderate', 'low', or 'very low'; this assessment will give the reader confidence that an estimate of effect or association reflects the item that is being assessed.

\section{ACKNOWLEDGEMENTS}

\section{RE F E R E N C E S}

\section{Additional references}

\section{ACT 2009}

Association for Children's Palliative Care.

Children's palliative care definitions. http://

www.togetherforshortlives.org.uk/assets/0000/1638/CPC_ definitions.pdf (accessed 20 June 2012).

\section{Anderson 2008}

Anderson BJ. Paracetamol (acetaminophen): mechanisms of action. Pediatric Anesthesia 2008;18(10):915-21.
Ballentine 2012

Ballantine N, Daglish EB. Chapter 17: Using medications in children. In: Goldman A, Hain R, Liben S editor(s). Oxford Textbook of Palliative Care for Children. 2nd Edition. Oxford: Oxford University Press, 2012:234-246.

Beretta 2010

Beretta S, Polastri D, Clerici CA, Casanova M, Cefalo $\mathrm{G}$, Ferrari A, et al.End of life in children with cancer: experience at the Pediatric Oncology Department of the Istituto Nazionale Tumori in Milan. Pediatric Blood \& 
Cancer 2010;54(1):88-91.

BNF 2012

Paediatric Formulary Committee. British National Formulary for Children 2012-2013. London: British Medical Association, the Royal Pharmaceutical Society of Great Britain, the Royal College of Paediatrics and Child Health, and the Neonatal and Paediatric Pharmacists Group, July 2012.

\section{Bouwmeester 2004}

Bouwmeester NJ, Anderson BJ, Tibboel D, Holford NHG. Developmental pharmacokinetics of morphine and its metabolites in neonates, infants and young children. British Journal of Anaesthesia 2004;92:208-17.

\section{Bradshaw 2005}

Bradshaw G, Hinds PS, Lensing S, Gattuso JS, Razzouk BI. Cancer-related deaths in children and adolescents. Journal of Palliative Medicine 2005;8(1):86-95.

Brook 2012

Brook L, Aindow A, Jassel S. Prescribing in paediatric palliative care: an association for paediatric palliative medicine survey. BMJ Supportive \& Palliative Care 2012; Vol. 2, issue Suppl 1:A24.

\section{Cochrane 2007}

Cochrane H, Liyanage S, Nantambi R. Palliative Care Statistics for Children and Young Adults. Health and Care Partnerships Analysis. London: Department of Health, 2007.

\section{Cochrane 2011}

Cochrane Pain, Palliative \& Supportive Care Review Group. Authoring or Assessing a Cochrane Protocol, Review, or Review Update for the PaPaS Review Group [updated September 2011]. http:// papas.cochrane.org/sites/papas.cochrane.org/files/uploads/ L\%20-\%20PaPaSAuthor\%26RefereeGuidance.pdf (accessed 19 July 2013).

\section{Drake 2003}

Drake R, Frost J, Collins JJ. The symptoms of dying children. Journal of Pain and Symptom Management 2003; 26(1):594-603.

\section{Feudtner 2011}

Feudtner C, Kang TI, Hexem KR, Friedrichsdorf SJ, Osenga K, Siden H, et al.Pediatric palliative care patients: a prospective multicenter cohort study. Pediatrics. 2011;127 (6):1094-101.

Fraser 2012

Fraser LK, Miller M, Hain R, Norman P, Aldridge J, McKinney PA, et al.Rising national prevalence of lifelimiting conditions in children in England. Pediatrics 2012; 129(4):e923-e9.

\section{Goldman 2006}

Goldman A, Hewitt M, Collins GS, Childs M, Hain R. Symptoms in children/young people with progressive malignant disease: United Kingdom children's cancer study group/paediatric oncology nurses forum survey. Pediatrics 2006;117(6):e1179-e86.

\section{Hain 2010}

Hain RDW, Devins M, Hastings R, Noyes J. Directory of life-limiting diagnoses in children, based on ICD-10 codes. Unpublished work on behalf of Together for Short Lives, 2010.

Hain 2011

Hain R, Ballantine N. Children: different- but the same species. European Journal of Palliative Care 2011;18(6): 288-92.

Hechler 2008

Hechler T, Blankenburg M, Friedrichsdorf SJ, Garske D, Hubner B, Menke A, et al.Parents' perspective on symptoms, quality of life, characteristics of death and endof-life decisions for children dying from cancer. Klinische Padiatrie 2008;220(3):166-74.

\section{Higgins 2011a}

Higgins JPT, Altman DG, Sterne JAC, on behalf of the Cochrane Statistical Methods Group and the Cochrane Bias Methods Group. Chapter 8. Assessing risk of bias in included studies. In: Higgins JPT, Green S editor(s). Cochrane Handbook for Systematic Reviews of Interventions Version 5.1.0. www.cochrane-handbook.org. The Cochrane Collaboration, 2011.

\section{Higgins 2011b}

Higgins JPT, Deeks JJ, Altman DG, on behalf of the Cochrane Statistical Methods Group. Chapter 16. Special topics in statistics. In: Higgins JPT, Green S editor(s). Cochrane Handbook for Systematic Reviews of Interventions Version 5.1.0. www.cochrane-handbook.org. The Cochrane Collaboration, 2011.

\section{IASP 2012}

International Association for the Study of Pain. Taxonomy. http://www.iasp-pain.org/Content/NavigationMenu/ GeneralResourceLinks/PainDefinitions/default.htm (accessed 15 September 2012).

\section{Jacqz-Aigrain 2006}

Jacqz-Aigrain E, Anderson BJ. Pain control: non-steroidal anti-inflammatory agents. Seminars in Fetal and Neonatal Medicine 2006;11(4):251-9.

\section{Jalmsell 2006}

Jalmsell L, Kreicbergs U, Onelöv E, Steineck G, Henter J-I. Symptoms affecting children with malignancies during the last month of life: a nationwide follow-up. Pediatrics 2006; 117(4):1314-20.

\section{Liberati 2009}

Liberati A, Altman DG, Tetzlaff J, Mulrow C, Gøtzsche PC, Ioannidiset JPA, et al.The PRISMA statement for reporting systematic reviews and meta-analyses of studies that evaluate health care interventions: explanation and elaboration. Annals of Internal Medicine 2009;151:4.

\section{Mashayekhi 2009}

Mashayekhi SO, Ghandforoush-Sattari M, Routledge PA, Hain RD. Pharmacokinetic and pharmacodynamic study of morphine and morphine 6-glucuronide after oral and intravenous administration of morphine in children with 
cancer. Biopharmaceutics \& Drug Disposition 2009;30(3): 99-106.

\section{McGrath 2008}

McGrath PJ, Walco G, Turk DC, Dworkin RH, Brown MT, Davidson $\mathrm{K}$, et al.Core outcome domains and measures for pediatric acute and chronic/recurrent pain clinical trials: PedIMMPACT recommendations. Journal of Pain 2008;9: $771-83$.

Ravens-Sieberer 2010

Ravens-Sieberer U, Wille N, Badia X, Bonsel G, Burström, $\mathrm{K}$, Cavrini $\mathrm{G}$, et al.Feasibility, reliability, and validity of the EQ-5D-Y: results from a multinational study. Quality of Life Research 2010;19(6):887-97.

Schunemann 2008

Schunemann HJ, Oxman AD, Brozek J, Glasziou P, Bossuyt P, Chang S, et al.GRADE: assessing the quality of evidence for diagnostic recommendations. Evidence-Based Medicine 2008;13(6):162-3.

Stinson 2006

Stinson JN, Kavanagh T, Yamada J, Gill N, Stevens B. Systematic review of the psychometric properties, interpretability and feasibility of self-report pain intensity measures for use in clinical trials in children and adolescents. Pain 2006;125(1-2):143-57.

Varni 1999

Varni JW, Seid M, Rode CA. The PedsQL (TM): measurement model for the pediatric quality of life inventory. Medical Care 1999;37(2):126-39.
Von Baeyer 2009

Von Baeyer CL, Spagrud LJ, McCormick JC, Choo E, Neville K, Connelly MA. Three new datasets supporting use of the Numerical Rating Scale (NRS-11) for children's self-reports of pain intensity. Pain 2009;143:223-7.

\section{WHO 1996}

World Health Organization. Cancer Pain Relief, Second Edition, With a Guide to Opioid Availability. Geneva: World Health Organisation, 1996.

\section{WHO 2012}

World Health Organisation. WHO Guidelines on Pharmacological Treatment of Persisting Pain in Children With Medical Illnesses. Geneva: World Health Organisation, 2012.

Wolfe 2000

Wolfe J, Grier HE, Klar N, Levin SB, Ellenbogen JM, Salem-Schatz $S$, et al.Symptoms and suffering at the end of life in children with cancer. New England Journal of Medicine 2000;342(5):326-33.

\section{Wong 1988}

Wong D. Baker C. Pain in children: comparison of assessment scales. Pediatric Nursing 1988;14(1):9-17.

\section{Zernikow 2009}

Zernikow B, Michel E, Craig F, Anderson BJ. Pediatric palliative care: use of opioids for the management of pain. Pediatric Drugs 2009;11(2):129-51.

* Indicates the major publication for the study

\section{A P P E N D I C ES}

\section{Appendix I. Medline search strategy}

1 exp Pain/

2 Pain Management/

3 (pain* or headache* or migraine* or neuralgia or neuropathic).mp.

4 or $/ 1-3$

5 exp Analgesics/

6 Anesthesia, Local/

7 exp Anticonvulsants/

8 exp Antidepressive Agents/

9 exp Anti-Inflammatory Agents, Non-Steroidal/

10 exp Muscle Relaxants, Central/

11 exp Parasympatholytics/

12 exp Serotonin Uptake Inhibitors/

13 exp Steroids/

14 (acetaminophen or "acetylsalicylic acid" or "alendronic acid" or alfentanil or amitriptyline or aspirin or baclofen or benzocaine or bupivacaine or buprenorphine or butorphanol or carbamazepine or chloroprocaine or "choline magnesium trisalicylate" or clonazepam or clonidine or codeine or dexamethasone or dexmetetomidine or dextroamphetamine or dextropropoxyphene or diamorphine or 
diazepam or diclofenac or dihydrocodeine or domperidone or fentanyl or fluoxetine or gabapentin or hydrocodone or hydromorphone or "hyoscine hydrobromide" or ibuprofen or ketamine or ketoprofen or ketorolac or "levo bupivacaine" or lidocaine or loperamide or lorazepam or mefenamic acid or meperidine or methadone or methylphenidate or midazolam or morphine or naproxen or nitrous oxide or nortriptyline or oxycodone or pamidronate or paracetamol or paroxetine or pentazocine or pethidine or phenobarbital or "phenytoin" or piroxicam or pregabalin or propoxyphene or "risedronate sodium" or "sodium clodronate" or tetracaine or tramadol or "valproic acid").mp.

15 exp Infant/

16 exp Child/

17 Adolescent/

18 (neonate* or newborn or infant* or child* or adolescen* or paediatric* or pediatric* or baby or babies or toddler* or teen* or juvenile* or boy* or girl*).mp.

19 or/ $15-18$

20 or $/ 5-14$

214 and 19 and 20

key:

$\mathrm{mp}=$ title, abstract, original title, name of substance word, subject heading word, protocol supplementary concept, rare disease supplementary concept, unique identifier

\section{CONTRIBUTIONSOFAUTHORS}

\begin{tabular}{|c|c|}
\hline Draft the protocol & All \\
\hline Develop a search strategy & All \\
\hline Search for studies (usually 2 review authors) & EB/JL (HR) \\
\hline Obtain copies of studies & EB \\
\hline Select which studies to include $(2+1$ arbiter $)$ & All \\
\hline Extract data from studies (2 review authors) & EB/JL (HR) \\
\hline Enter data into RevMan & EB \\
\hline Carry out the analysis & EB \\
\hline Interpret the analysis & All \\
\hline Draft the final write-up of the review & EB \\
\hline Update the review & $\mathrm{EB} / \mathrm{LJ} / \mathrm{MB}-\mathrm{L}$ \\
\hline Serve as content expert & RH/JL/RM \\
\hline Take responsibility for grammar and language & LJ \\
\hline Serve as methodologist & $\mathrm{BC} / \mathrm{VV}$ \\
\hline
\end{tabular}

Copyright $\odot 2013$ The Cochrane Collaboration. Published by John Wiley \& Sons, Ltd. 


\section{DECLARATIONSOF INTEREST}

None known.

\section{SOURCES OF SUPPORT}

\section{Internal sources}

- Marie Curie Cancer Care core grant funding, UK.

Emma Beecham's post is funded by Marie Curie Cancer Care's core grant MCCC-FCO-11-U

\section{External sources}

- No sources of support supplied 